\title{
ADAPTACIÓN DE LENTES DE CONTACTO RÍGIDAS PERMEABLES AL GAS EN UN CASO DE GLAUCOMA CONGÉNITO
}

\section{RIGID GAS PERMEABLE CONTACT LENSES FITTED IN A CASE OF CONGENITAL GLAUCOMA}

\author{
PALOMO-ÁLVAREZ C ${ }^{1}$, MARTÍN-GARCÍA B ${ }^{2}$
}

\section{RESUMEN}

Caso clínico: Mujer de 27 años tratada de glaucoma congénito con alta miopía, baja AV y nistagmus en ambos ojos, fue referida para adaptar lentes de contacto. Se adaptan lentes de contacto rígidas permeables a los gases (LC RPG) asféricas y de alta transmisibilidad en uso diario. La paciente mejoró la AV con confort durante 10 horas al día de porte. Discusión: La adaptación de lentes de contacto se realizó teniendo en cuenta la topografía corneal de la paciente. Consideramos las lentes de contacto permeables al gas como las lentes de primera elección para estos pacientes, que con una adecuada adaptación y seguimientos pueden utilizar con seguridad lentes de contacto RPG en uso diario.

Palabras clave: Glaucoma congénito, alta miopía, agudeza visual baja, nistagmus, lentes de contacto rígidas permeables a los gases (LC RPG).

\begin{abstract}
Case report: A 27-year-old woman with congenital glaucoma with high myopia, low visual acuity and nystagmus in both eyes, was referred for contact lens fitting. We fitted her with aspherical rigid gas permeable (RGP) contact lenses with high transmissibility, which she wore on a daily basis. Her visual acuity improved with the wearing of these lenses, which remained comfortable whilst being worn for 10 hours a day.

Discussion: Contact lenses were fitted according to her corneal topography. Gas permeable contact lenses were chosen as first choice and with proper fitting and vigilant follow-up they may be worn safely on a daily basis (Arch Soc Esp Oftalmol 2008; 83: 377-380).
\end{abstract}

Key words: Congenital glaucoma, high myopia, low visual acuity, nystagmus, rigid gas permeable contact lenses (RGP CL).

\footnotetext{
Recibido: 29/10/07. Aceptado: 24/4/08.

Escuela Universitaria de Óptica. Universidad Complutense de Madrid. Madrid. España.

1 Licenciado en Ciencias de la Educación. Diplomado en Óptica y Optometría.

2 Diplomado en Óptica y Optometría. 


\section{INTRODUCIÓN}

En el Glaucoma Congénito Primario la elevación patológica de la PIO produce la distensión de tejidos oculares con el resultado de la aparición de edema corneal, estrías, opacidades corneales y alteración de distintas estructuras oculares; así como, miopía progresiva debido a la elasticidad escleral (1). La aparición de un nistagmus, es poco frecuente en el glaucoma congénito y su existencia es la expresión de un déficit visual grave.

El uso de lentes de contacto rígidas permeables a los gases (LRPG) es la primera elección en pacientes que presentan miopías elevadas y/o con irregularidades corneales, ya que aportan una mayor calidad óptica y seguridad en el uso. No existe contraindicación directa en pacientes poliintervenidos de glaucoma congénito en uso estrictamente diario $(2,3)$; no obstante no existen muchas investigaciones en este campo.

\section{CASO CLÍNICO}

Mujer de 27 años remitida desde el servicio de oftalmología del Hospital Clínico San Carlos de Madrid (Junio de 2006) diagnosticada y tratada de glaucoma congénito desde el nacimiento, para adaptar lentes de contacto. La paciente también presenta nistagmus.

En la historia ocular la paciente refiere cuatro cirugías en cada ojo desde el nacimiento hasta los diez años y posteriormente implante de derivación a los 18 y 21 años en ambos ojos.

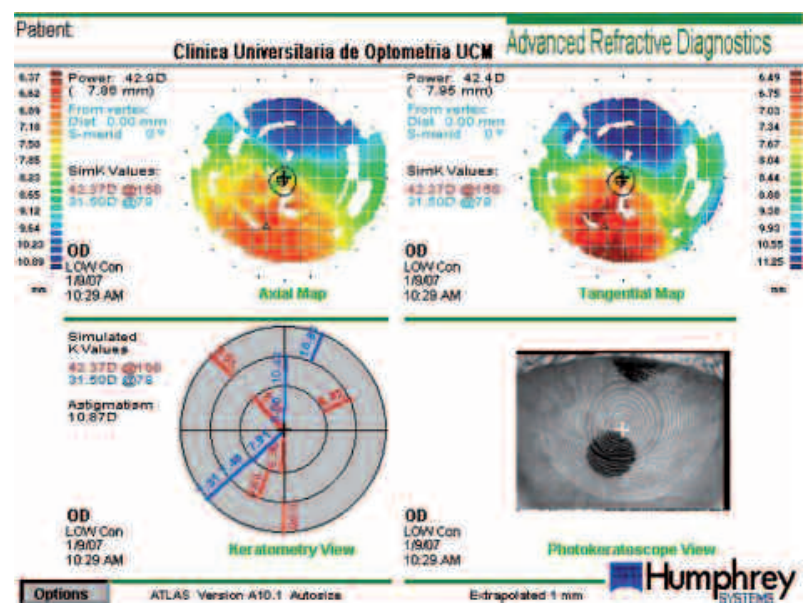

Actualmente, continúa con tratamiento farmacológico en OD y lubricantes oculares en AO.

La paciente porta compensación en gafa de $-11.75 \mathrm{D}$ en $\mathrm{AO}$ con la que alcanza AV de 0.05 y 0.16 en OD y OI respectivamente. Realizamos refracción subjetiva y con ésta:

OD: -14.00 D y OI: -14.00 - 1.00 a $90^{\circ}$ alcanza una AV menor de 0.05 y 0.2 + para cada ojo respectivamente.

La topografía corneal muestra irregularidades corneales en ambos ojos (fig. 1. a y b).

Las pupilas presentan asimetría, alteraciones de forma, posición descentrada y diámetros medios de 3 y $6 \mathrm{~mm}$ respectivamente en OD y OI.

La evaluación biomicroscópica de la córnea evidencia disminución de la transparencia corneal, mala calidad lagrimal presentando un tiempo de rotura lagrimal de 2 segundos, y tinción difusa con fluoresceína mas localizada en el tercio inferior de córnea. El diámetro corneal es de 16 mm en ambos ojos.

Después de valorar los resultados de la evaluación se decidió comenzar la adaptación con LRPG. Se realizaron varias pruebas y las lentes definitivas que se adaptan son lentes RPG Confort (Contamac ${ }^{\circledR}$, UK) de diseño asférico (AZV Oxicon Confort) del laboratorio Lenticon ${ }^{\circledR}$ (tabla I y fig. 2). Este material está formado por combinación de polímeros de fluorsilicona de alta pureza con un componente hidrofílico y DK (Fatt modificado) de 60.

La AV que alcanza la paciente es para el OD: 0,05, OI: 0,3+1 y binocular: 0,4 -2.

El porte de las lentes es progresivo hasta alcanzar un máximo entre 8 y 10 horas, siempre en régimen de uso diario. La paciente refiere inicialmente difi-

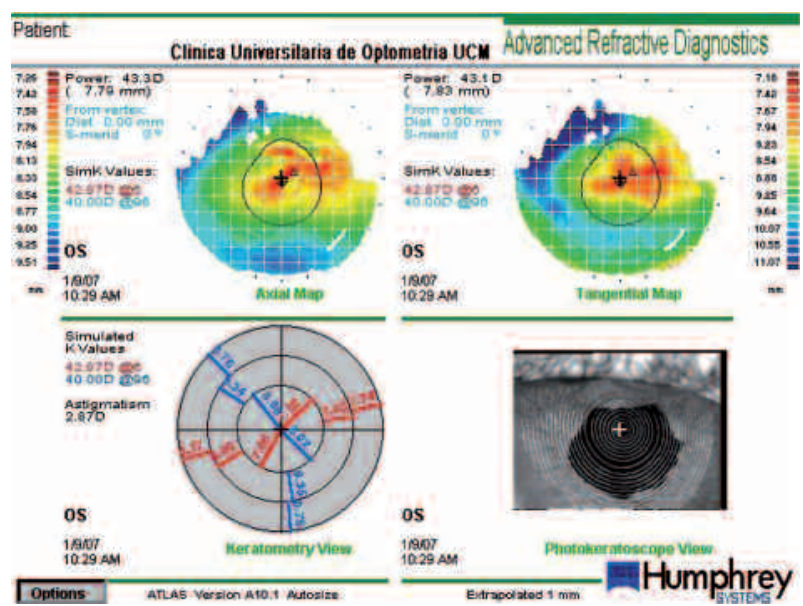

Fig. 1: Topografía de ojo derecho (a) e izquierdo (b) de la paciente. 
Tabla I. Parámetros de las lentes de contacto adaptadas

\begin{tabular}{lcccc}
\hline & Radio base $(\mathrm{mm})$ & Potencia $(\mathrm{D})$ & Diámetro total $(\mathrm{mm})$ & Excentricidad \\
\hline L. Derecha & 7,35 & $-13,50$ & 10,00 & 0,6 \\
L. Izquierda & 8,25 & $-12,00$ & 10,00 & 0,3 \\
\hline \hline
\end{tabular}
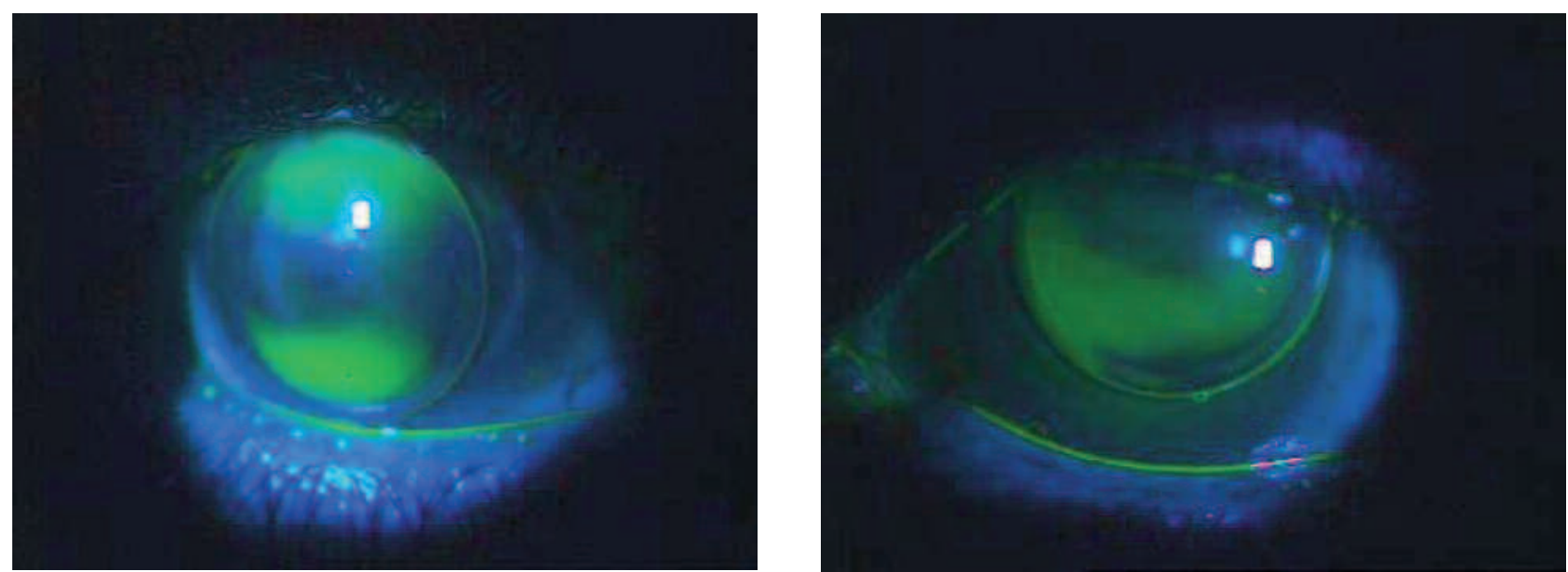

Fig. 2: Fluorograma de las lentes de contacto adaptadas en $O D(a)$ y OI (b).

cultades en visión próxima con las lentes de contacto desde el inicio de la adaptación hasta el mes de porte habitual. Esto es debido a que en un ojo miope la convergencia y acomodación que se requiere con lentes de contacto es mayor a la que se emplea con gafas (2).

En octubre de 2006 la paciente sufre una caída que produce traumatismo con desprendimiento coroideo de OI, requiriendo nueva intervención en este ojo. Una vez tratada, en enero de 2007 se procede a la reposición de las lentes perdidas durante la caída. Las alteraciones sufridas en el OI requieren ligeras modificaciones en la potencia $(-13,00 \mathrm{D}) \mathrm{y}$ la excentricidad $(0,4)$ de la lente izquierda, consiguiendo una $\mathrm{AV}$, tras 4 meses de uso de las lentes, de 0,05 en OD, 0,3-2 en OI y binocular: 0,3 -2.

Durante el proceso de la adaptación su OI presenta tinción localizada en zona inferonasal, sin causa mecánica por parte de la lente de contacto (fig. 3). La lesión no remite suspendiendo el porte de las lentes. La paciente fue remitida al oftalmólogo que diagnosticó la lesión como una úlcera trófica, iniciándose tratamiento con suero autólogo que resolvió la lesión al mes de tratamiento (3). Posteriormente continúa con el uso de las lentes de contacto en régimen de uso diario.

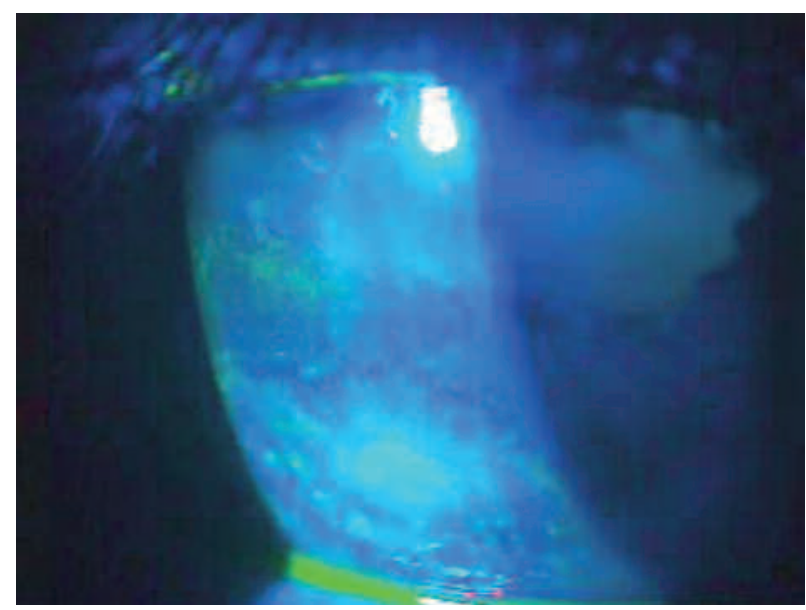

Fig. 3: Tinción con fluoresceína del OI.

\section{DISCUSIÓN}

El proceso de adaptación de lentes de contacto permeables al gas en este caso se realiza en base a la topografia corneal. La tolerancia de las LRPG en este caso es buena y la paciente aprecia mejoría en la calidad visual.

No hay muchas investigaciones, y por tanto contraindicaciones, sobre las adaptaciones de lentes de 
contacto RPG en pacientes con glaucoma congénito, aunque, sí es necesario un mayor control ante posibles infecciones $(4,5)$. Actualmente tenemos una gran variedad en diseños y materiales de LC RPG que permiten una adaptación y porte seguros, por lo cual, las consideremos como lentes de primera elección recomendando seguimientos frecuentes.

\section{BIBLIOGRAFÍA}

1. Freedman SF, Walton DS. Glaucoma in infants and children. In: Nelson LB, Olitsky SE. Harley's Pediatric Opht- halmology. Philadelphia: Lippincott Williams \& Wilkins. 2005; 285-304.

2. Lois N, Dias JL, Cohen EJ. Use of contact lenses in patients with filtering blebs. CLAO Journal 1997; 23: 100-102.

3. Samples JR, Andre M, MacRae SM. Use of gas permeable contact lenses following trabeculectomy. CLAO Journal 1990; 16: 282-284.

4. Ford MW, Stone J. Practical optics and computer design of contact lenses. In: Phillips AJ, Speedwell L. Contact lenses. Oxford: Butterworth-Heinemann; 1997; 154-231.

5. Alvarado Valero MC, Martínez Toldos JJ, Borras Blasco $J$, Almiñana Almiñana A, Pérez Ramos JM. Tratamiento de defectos epiteliales persistentes mediante suero autólogo. Arch Soc Esp Oftalmol 2004; 79: 537-542. 